Reprod. Nutr. Dévelop., 1981, 21 (4), 513-518.

\title{
Recherche de phosphopeptides dans les fèces de rats axéniques alimentés avec de la caséine ovine radioactive
}

\author{
par J. P. PELISSIER, Françoise DUBOS *, F. DABURON ** \\ Laboratoire de Biochimie ef Technologie Laitières \\ * Laboratoire d'Ecologie Microbienne \\ ** Laboratoire de Radiobiologie Appliquée, C.E.A. \\ I.N.R.A., 78350 jouy en Josos, France.
}

Summary. Search for phosphopeptides in the feces of axenic rats fed radioactive ovine casein.

Radioactive ovine casein was obtained by injecting $100 \mu \mathrm{Ci}$ of ${ }^{14} \mathrm{C}$-Ser into the jugular vein of an ewe. The milk collected 17 and $24 \mathrm{~h}$ after this injection contained $12 \mathrm{p} .100$ of the radioactivity injected in protein form. The seryl residues were specificially labelled.

This casein was used as the only protein source fed to axenic rats; 0.30 p. 100 of the tracer ingested was found in the feces of those rats. Since phosphoserine represented $25 \mathrm{p}$. 100 of the total casein seryl residues, the phosphopeptides may not be selectively unabsorbable.

\section{Introduction.}

La détermination des structures primaires d'un certain nombre de caséines a permis de mettre en évidence la nature des sites de phosphorylation (Ribadeau-Dumas, 1979) ainsi que l'existence d'une séquence de 6 résidus d'acides aminés, dont plusieurs sont phosphorylés, commune aux caséines sensibles au calcium $\alpha_{s 1}, \alpha_{s 2}$ et $\beta$. Le rôle essentiel de ces phosphoprotéines est probablement d'assurer la nutrition proféique du nouveau-né. Cependant, la conservation d'un segment de leur séquence au cours de l'évolution (fig. 1) pourrait s'expliquer par un rôle physiologique, autre que celui d'apport d'azote ou d'acides aminés. Cette fonction aurait produit une pression de sélection et ainsi maintenu cet enchainement d'acides aminés dans les différentes caséines provenant de diverses espèces.

-ILE-SERP-SERP-SERP-GLU-GLU-VAL-SERP-SERP-SERP-GLU-GLU-GLY-SERP-SERP-SERP-GLU-GLU-LEU-SERP-THR-SERP-GLU-GLU-LEU-SERP-SERP-SERP-GLU-GLU-

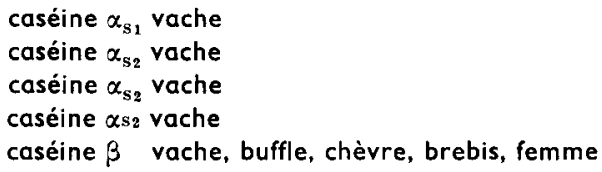

FIG. 1. - Structure du fragment phosphorylè des caséines.

Naito et Suzuki (1974), postulant pour ces phosphopeptides un rôle dans le transport du calcium dans le tube digestif, ont mis en évidence leur présence dans le tiers 
distal de l'intestin grêle du rat. Leur structure se révèle donc résistante à l'action des diverses protéases et phosphatases du système digestif et une dégradation tardive et complète après ce stade du transit intestinal apparaît peu probable. Ces phosphopeptides, dont la taille semble exclure une absorption directe par la muqueuse intestinale, devraient donc se retrouver intacts dans les fèces en fin de digestion. Le but de ce travail était donc de vérifier si ces phosphopeptides sont effectivement présents dans les fèces.

Un marquage spécifique des phosphopeptides est nécessaire pour les repérer dans les fèces, L'utilisation de caséine radioactive obtenue in vivo constitue un moyen de différencier l'azote exogène de l'azote endogène. Afin de repérer plus spécifiquement les phosphopeptides, le marqueur utilisé peut, soit être porté par les groupements phosphates, mais l'utilisation du ${ }^{32} \mathrm{P}$ est délicale pour des manipulations longues sur animaux, soit être porté par les résidus d'acides aminés sur lesquels sont fixés ces groupements, dans notre cas les résidus séryles. Les phosphosérines représentant 25 p. 100 de l'ensemble des résidus séryles des caséines, ce marquage est donc relativement spécifique des phosphopeptides.

De plus, l'utilisation d'animaux axéniques permet de déterminer si, en l'absence de flore bactérienne, le tube digestif de l'animal est capable, à lui seul, de transformer ces peptides.

Au cours du présent travail, nous avons préparé de la caséine ovine entière dont les résidus séryles étaient marqués au ${ }^{14} \mathrm{C}$. La caséine a été utilisée comme seule source d'azote pour nourrir des rats axéniques.

\section{Matériel et méthodes.}

- La caséine radioactive a été obtenue par injection de $100 \mu \mathrm{Ci}$ de ${ }^{14} \mathrm{C}$-sérine dans la jugulaire d'une brebis (race française, Préalpes), juste après la traite du soir. Le lait était recueilli le lendemain matin, $17 \mathrm{~h}$ après l'injection, et le lendemain soir, $24 \mathrm{~h}$ après l'injection. La caséine a été obłenue après écrémage, par précipitation du lait de la première traite à $\mathrm{pH} 4,6$, avec $\mathrm{HCl} \mathrm{N}$. Elle a été ensuite mise en solution dans de l'eau maintenue à $\mathrm{pH} 7,0$ par adjonction de $\mathrm{NaOH} \mathrm{N}$, puis lyophilisée. Les protéines totales ont été obtenues par dialyse puis lyophilisation du lait écrémé.

- La séparation des différentes caséines ovines a été effectuée par chromatographie sur DEAE-cellulose selon Mercier ef al. (1968). Chaque fraction obtenue a été caractérisée par électrophorèse sur gel d'amidon selon Schmidt (1974).

- Les animaux utilisés étaient des rats axéniques (Fisher) adultes maintenus en isolateurs. Ils étaient nourris avec un régime solide ayant, pour seule source de protéine, la caséine entière marquée. Ce régime (łabl. 1), stérilisé par aułoclavage, éłait fourni ad libifum à deux rats axéniques. Les fèces des animaux, placés sur grille, étaient récupérées chaque jour.

- Les fèces ont été extraites par de l'acide acétique 30 p. 100 ef la fraction soluble a été chromatographiée sur Sephadex G25 (Pharmacia, Uppsala) dans le même solvant. - Un fractionnement par chromatographie d'échange d'ions a été réalisé sur résine Dowex AG 50W-X2, livrée dans le commerce sous forme $\mathrm{H}^{+}$, et qui avait subi les traitements préconisés par Moore et Stein (1951). Avant utilisation, la résine était traitée 
TABLEAU 1

Composition du régime alimentaire des rats (par $\mathrm{kg}$ d'aliment)

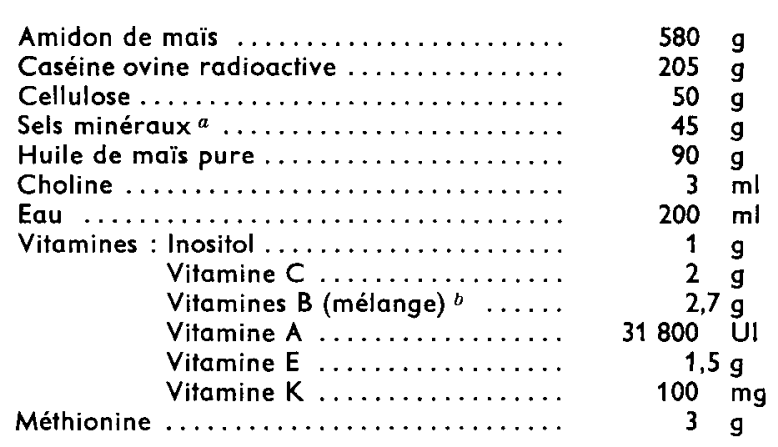

$a$ Les sels minéraux contiennent : $\mathrm{CaCO}_{3}(15 \mathrm{~g}) ; \mathrm{K}_{2} \mathrm{HPO}_{4}(11,25 \mathrm{~g}) ; \mathrm{CaHPO}_{4}(2,75 \mathrm{~g}) ; \mathrm{Na}_{2} \mathrm{HPO}_{4}$ $(10 \mathrm{~g}) ; \mathrm{NaCl}(2,5 \mathrm{~g}) ; \mathrm{MgSO}_{4}, 7 \mathrm{H}_{2} \mathrm{O}(3,75 \mathrm{~g})$; Citrate de fer $(3,75 \mathrm{~g}) ; \mathrm{MnSO}_{4}(625 \mathrm{mg}) ; \mathrm{KI}(37,5 \mathrm{mg})$; $\mathrm{CoCl}_{2}, 6 \mathrm{H}_{2} \mathrm{O}(25 \mathrm{mg}) ; \mathrm{ZnSO}_{4}, 7 \mathrm{H}_{2} \mathrm{O}(50 \mathrm{mg})$.

$b$ Le mélange de vitamines $\mathrm{B}$ contient : Thiamine $(150 \mathrm{mg})$; Riboflavine $(75 \mathrm{mg})$; Nicotinamide $(125 \mathrm{mg})$; Acide nicotinique $(125 \mathrm{mg})$; Pantothénate de calcium (750 mg) ; Pyridoxine (100 mg) ; Biotine (2,5 mg) ; Acide folique $(25 \mathrm{mg})$; Acide para-aminobenzoïque (125 mg) ; Vitamine B12 (625 mg).

selon Schroeder et al. (1962) ; au stade final, elle était mise en suspension dans un tampon pyridine-acide acétique-eau, $\mathrm{pH} 3,1,0,2 \mathrm{~N}$ en pyridine $(64,5: 1114: 2821,5$, $\mathrm{v} / \mathrm{v} / \mathrm{v}$ ). Après élution par ce tampon, un tampon similaire de $\mathrm{pH} 5,3,4 \mathrm{~N}$ en pyridine (1 $290: 400: 2310, \mathrm{v} / \mathrm{v} / \mathrm{v}$ ) était utilisé pour éluer les peptides fixés sur la résine. Enfin, de la soude $0,2 \mathrm{~N}$ a permis de récupérer les produits encore fixés sur la résine.

- Le dosage des acides aminés était effectué à l'aide d'un analyseur Beckman « Multichrom », après hydrolyse de $24 \mathrm{~h}$ à $110^{\circ} \mathrm{C}$ dans des tubes scellés sous vide, en présence de $\mathrm{HCl} 5,7 \mathrm{~N}$ redistillé trois fois.

- Les électrophorèses à haute tension sur papier étaient réalisées à l'aide d'un appareil Savant (Hicksville, N.Y.), en tampon pH 4,7 (pyridine-acide acétique-eau, $2: 2$ : $96 \mathrm{v} / \mathrm{v} / \mathrm{v})$. La tension appliquée était de l'ordre de $60 \mathrm{~V} / \mathrm{cm}$; la durée des électrophorèses était de $30 \mathrm{~min}$. Les produits étaient ensuite révélés par trempage du papier dans un réactif ninhydrine-collidine $(1 \mathrm{~g}$ de ninhydrine, $50 \mathrm{ml}$ de collidine, $980 \mathrm{ml}$ d'éthanol). - La radioactivité a été mesurée dans un compteur à scintillation Packard 3375 ; $8,5 \mathrm{ml}$ de liquide scintillant : Triton-Toluène-2-5 diphenyloxazole-1,4 bis-2 (4 méthyl 5 phényloxazolyl) benzène $(500 / 1000 / 4 / 0,1 ; \mathrm{v} / \mathrm{v} / \mathrm{g} / \mathrm{g})$ étaient ajoutés à l'échantillon $(2,5 \mathrm{ml})$.

\section{Résultats.}

Marquage de la caséine. - La première traite, $17 \mathrm{~h}$ après l'injection, a permis d'obtenir $570 \mathrm{ml}$ de lait qui ont fourni $32,6 \mathrm{~g}$ de caséine et $5,2 \mathrm{~g}$ de protéines du lactosérum contenant respectivement 8,7 et 1 p. 100 de la radioactivité injectée à l'animal. La traite effectuée $24 \mathrm{~h}$ après l'injection a fourni $19,5 \mathrm{~g}$ de protéines totales du lait contenant 2,3 p. 100 de la radioactivité injectée. 
La sérine n'étant pas un acide aminé indispensable, nous avons vérifié, par hydrolyse acide de la caséine entière, séparation des différents acides aminés par l'analyseur et mesure de leur radioactivité, que la sérine était le seul acide aminé marqué. Afin de vérifier que chacune des caséines contenait de la radioactivité, nous avons chromatographié sur une colonne de DEAE-cellulose un mélange de $600 \mathrm{mg}$ de caséine ovine radioactive et de $1,4 \mathrm{~g}$ de caséine ovine froide. L'élution a été suivie par mesure de la densité optique à $280 \mathrm{~nm}$ et de la radioactivité (fig. 2). Le profil d'élution correspond à celui obtenu classiquement avec les caséines ovines (Mercier et al., 1968). Chaque pic a été identifié par électrophorèse sur gel d'amidon.

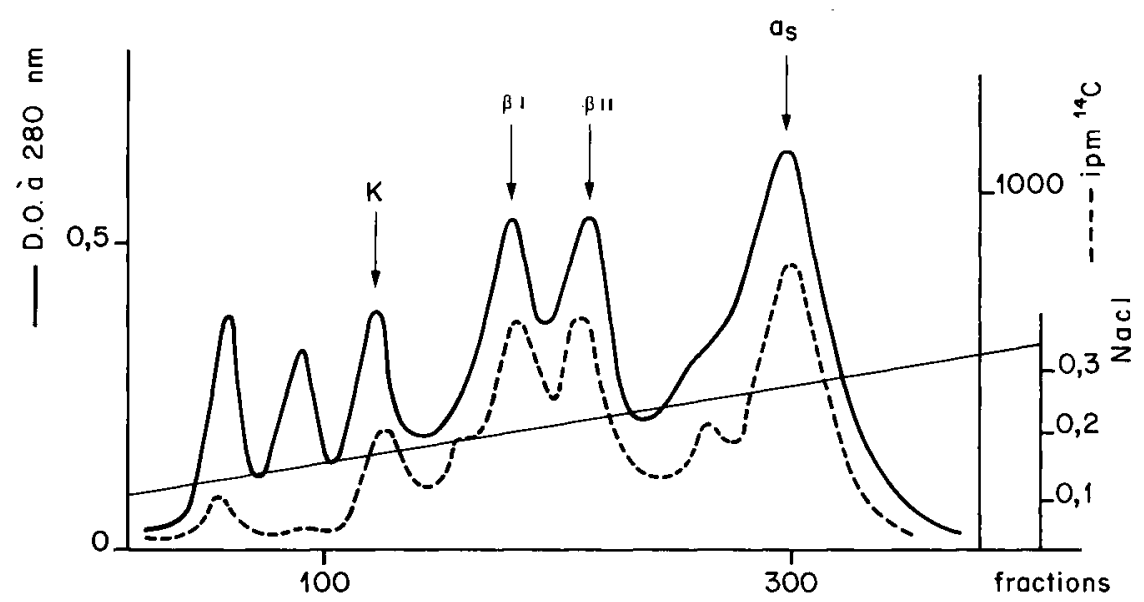

FIG. 2. - Diagramme d'élution des caséines ovines sur DEAE-cellulose. Diamètre : $3 \mathrm{~cm} ;$ hauteur : $20 \mathrm{~cm}$; fraction : $10 \mathrm{ml}$; débit : $200 \mathrm{ml} / \mathrm{h}$. Charge : $0,6 \mathrm{~g}$ de caséine radioactive $+1,4 \mathrm{~g}$ de caséine froide. Elution par tampon urée $3,3 \mathrm{M}$; imidazole $0,02 \mathrm{M}, \mathrm{pH} 7,0$, additionné de 2-mercaptoéthanol (1 $\mathrm{ml} / \mathrm{l})$. Elution par un gradient de $\mathrm{NaCl}$ de 0 à $0,35 \mathrm{M}(2 \mathrm{l} / 2 \mathrm{l})$.

Etude du contenu des fèces. - La radioactivité obtenue chaque jour dans les fèces est reportée dans le tableau 2.

TABLEAU 2

Rodiooctivité récupérée par rat dans les fèces

\begin{tabular}{|c|c|c|c|c|c|}
\hline & \multirow{2}{*}{$\begin{array}{l}\text { Quantifé } \\
\text { ingérée }\end{array}$} & \multicolumn{4}{|c|}{ Fèces } \\
\hline & & $1^{\mathrm{er}}$ jour & $2 \mathrm{e}$ jour & $3^{e}$ jour & $4 \mathrm{e}$ jour \\
\hline Poids (g) $\ldots \ldots \ldots \ldots \ldots \ldots$ & 22 & 2,6 & 4,8 & 4,9 & 7,4 \\
\hline $\mathrm{ipm} / \mathrm{g} \ldots \ldots \ldots \ldots \ldots \ldots \ldots$ & 73800 & 70 & 130 & 490 & 200 \\
\hline ipm totales ................ & 1620000 & 190 & 630 & 2400 & 1500 \\
\hline p. 100 cumulé de radioactivité . & 100 & 0,01 & 0,05 & 0,20 & 0,29 \\
\hline
\end{tabular}


Au total, la radioactivité récupérée en 4 jours dans les fèces ne représente qu'une faible part de la radioactivité ingérée, environ 0,30 p. 100. La fraction insoluble dans l'acide acétique 30 p. 100 ne présentant pas de radioactivité, seule la fraction soluble a été analysée. Par chromatographie sur Sephadex G25, 2 fractions A et B ont été obtenues (fig. 3). Chacune d'elles contenait une radioactivité totale voisine.

La fraction B a été rechromatographiée sur la même colonne de Sephadex G25, ce qui a permis d'obtenir 2 fractions, B1 et B2 (fig. 4). Par électrophorèse sur papier à $\mathrm{pH}$ 4,7 de la fraction B1, la radioactivité migre au niveau des acides aminés neutres. Elle ne contenait donc pas de phosphopeptides. La quantité de radioactivité contenue dans cette fraction n'a pas permis de pousser plus loin sa purification.

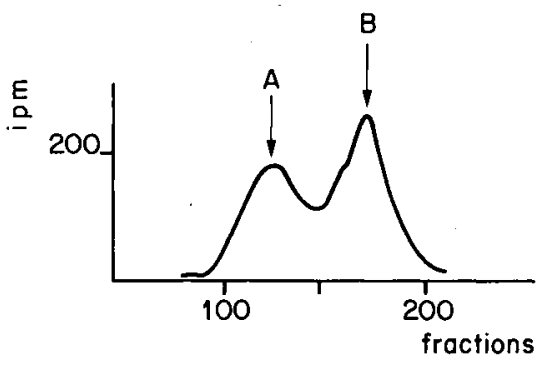

FIG. 3. - Diagramme d'élution sur Sephadex G25 de la fraction des fèces soluble dans l'acide acétique 30 p. 100 . Diamètre $: 3 \mathrm{~cm}$; hauteur : $175 \mathrm{~cm}$; fraction : $10 \mathrm{ml}$; débit : $30 \mathrm{ml} / \mathrm{h}$. Elution par l'acide acétique 30 p. 100.

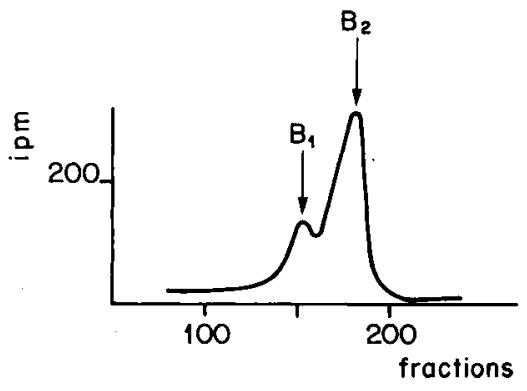

FIG. 4. - Diagramme d'élution sur Séphadex G25 de la fraction $B$ (mêmes conditions que pour la figure 3).

La fraction B2 a été purifiée sur Dowex 50 puis sur l'analyseur d'acides aminés. La radioactivité contenue dans cette fraction est uniquement constituée par de la sérine libre non phosphorylée.

La fraction A, rechromatographiée sur G25 puis sur Dowex 50 , s'est révélée hétérogène. La faible radioactivité de cette fraction n'a pas permis d'identifier ses composants, ni de conclure à l'absence de phosphopeptides.

\section{Discussion.}

- La radioactivité de la caséine ovine obtenue $17 \mathrm{~h}$ après l'injection de $100 \mu \mathrm{Ci}$ de sérine marquée (10000 dps/g) est comprise entre celles obtenues chez la vache par Mills (1976) lors des traites effectuées $7 \mathrm{~h}(16000 \mathrm{dps} / \mathrm{g})$ et $11 \mathrm{~h}(5200 \mathrm{dps} / \mathrm{g})$ après l'injection de $2 \mathrm{mCi}$ d'un mélange de 20 acides aminés radioactifs. La brebis semble donc un animal plus facile à utiliser pour ce genre d'expérimentation : animal de plus petite taille, donc moins onéreux ef plus facile à manipuler, quantité à injecter plus faible, quantité de lait obtenu notable.

Bien que nous ayons utilisé un acide aminé non essentiel, le marquage obtenu $17 \mathrm{~h}$ après l'injection est resté spécifique de l'acide aminé utilisé. Cela révèle la très grande vitesse d'utilisation des acides aminés libres du sang pour la synthèse des protéines du 
lait, vifesse qui ne laisse que peu de place pour un autre métabolisme chez la brebis allaitante. Une telle technique devrait donc permettre de marquer sélectivement tel ou tel type d'acide aminé dans la chaine protéique selon l'utilisation expérimentale ultérieure des protéines du lait.

- La radioactivité totale, récupérée en 4 jours dans les fèces, représente $0,30 p$. 100 de la radioactivité ingérée. De plus, près de la moitié de cette radioactivité est apportée par de la sérine libre (fraction B2). Dans le cas où les phosphopeptides ne seraient pas dégradés ou absorbés par l'intestin, la radioactivité obtenue devrait représenter 25 p. 100 de la radioactivité ingérée. Bien que nous ayons utilisé seulement deux rats pour cette expérience, l'écart observé est tel qu'il semble possible de conclure que les phosphopeptides ne sont pas sélectivement rejetés dans les fèces. Cependant, ces résultats mériteraient d'être confirmés par l'utilisation d'un plus grand nombre d'animaux et en suivant le devenir de ces peptides aux différents niveaux du tractus digestif. Naito et Suzuki (1974) ont mis en évidence, 1 h à 2 h 30 après l'ingestion d'un repas à base de caséine, la présence de peptides, riches en phosphore dans le tiers distal de l'intestin grêle du rat. Ces peptides ont une composition voisine de celle des phosphopeptides de caséine obtenu par action in vitro de la trypsine. Il serait donc intéressant de déterminer la nature et les quantités exactes de ces phosphopeptides qui apparaissent très résistants aux protéases ef phosphatases du système digestif.

Nous venons de montrer que les phosphopeptides ne sont probablement pas rejetés dans les fèces. L'utilisation d'animaux axéniques permet d'éliminer l'hypothèse de leur dégradation dans le gros intestin par la flore microbienne. Si les résultats de Naito et Suzuki sont exacts, cela entraîne que les phosphopeptides doivent être absorbés par l'intestin. Cette possibilité semble assez surprenante du fait de la taille de ces peptides (au moins 6 acides aminés). Une étude plus détaillée des transformations des phosphopeptides au cours du transit intestinal, et en particulier sous l'action des enzymes de la bordure en brosse de l'intestin, semble donc nécessaire afin de connaître leur devenir durant la digestion.

Reçu en octobre 1980.

Accepté en février 1981.

\section{Références}

MERCIER J. C., MAUBOIS J. L., POZNANSKI S., RIBADEAU-DUMAS B., 1968. Fractionnement préparatif des caséines de vache ef de brebis par chromatographie sur DEAE cellulose, en milieu urée et 2-mercaptoéthanol. Bull. Soc. Chim. biol., 50, 521-530.

MILLS O. E., 1976. The preparation of ${ }^{14} \mathrm{C}$-labelled milk proteins. N. Z. Dairy Sci. Technol., 11, 164-168.

MOORE S., STEIN W. H., 1951. Chromatography of amino acids on sulfonated polystyrene resins. J. biol. Chem., 192, 663-681.

NAITO H., SUZUKI H., 1974. Further evidence for the formation in vivo of phosphopeptides in the intestinal lumen from dietary $\beta$ casein. Agr. biol. Chem., 38, 1543-1545.

RIBADEAU.DUMAS B., 1979. Progrès récents dans la biochimie des protéines du lait. Rev. Lait. fr., 371, 1-15.

SCHMIDT D. G., 1974. Starch-gel electrophoresis of $x$ casein. Biochim. biophys. Acta, 90, 411-414.

SCHROEDER W. A., JONES R. I., CORMICK J., MC CALLA K., 1962. Chromatographic separation of peptides on ion exchange resins. Anal. Chem., 34, 1570-1575. 